\title{
Does an increase in energy return and/or longitudinal bending stiffness shoe features reduce the energetic cost of running?
}

\author{
Nicolas Flores $^{1,2}$ D $\cdot$ Nicolas Delattre $^{1} \cdot$ Eric Berton $^{2}$ D $\cdot$ Guillaume Rao $^{2}$ (D
}

Received: 3 August 2018 / Accepted: 17 November 2018

c) Springer-Verlag GmbH Germany, part of Springer Nature 2018

\begin{abstract}
Purpose This study focused on the effects of shoe energy return and shoe longitudinal bending stiffness on the energetic cost and biomechanics of running.

Methods The energetic cost of running and biomechanical variables altering running economy (ground contact times, stride frequency, vertical and leg stiffness, ground reaction force impulses, alignment between the resultant ground reaction force and the leg) were measured for nineteen male recreational runners. Participants ran overground under their ventilatory anaerobic threshold $\left(10.8 \pm 1.1 \mathrm{~km} \mathrm{~h}^{-1}\right.$ on average) using four shoe prototypes with features combining low or high magnitudes of energy return and longitudinal bending stiffness.

Results Neither the energy return, nor the longitudinal bending stiffness, or the interaction of these shoe features altered the energetic cost of running. High energy return shoes induced significant increased ground contact time from $274.5 \pm 18.3$ to $277.1 \pm 18.7 \mathrm{~ms}$, and significant decreased stride frequency from $1.34 \pm 0.05$ to $1.33 \pm 0.05 \mathrm{~Hz}$. High bending stiffness shoes induced significant increased ground contact time from $273.8 \pm 18.2$ to $277.9 \pm 18.7 \mathrm{~ms}$, significant increased vertical stiffness from $23.2 \pm 3.4$ to $23.8 \pm 3.0 \mathrm{kN} \mathrm{m}^{-1}$, and significant decreased net vertical impulse from $245.4 \pm 17.2$ to $241.7 \pm 17.5 \mathrm{BW} \mathrm{ms}$. Conclusions Increased energy return and longitudinal bending stiffness induced subtle changes in the running biomechanics, but did not induce any decrease in the energetic cost of running.
\end{abstract}

Keywords Footwear · Running economy $\cdot$ Biomechanics $\cdot$ Ground reaction force

$\begin{array}{ll}\text { Abbreviations } \\ \text { ANOVA } & \text { Analysis of variance } \\ \text { BW } & \text { Body weight } \\ \text { GRF } & \text { Ground reaction force } \\ \text { RE } & \text { Running economy } \\ \text { SPM } & \text { Statistical parametric mapping } \\ \text { VAT } & \text { Ventilatory anaerobic threshold }\end{array}$

Communicated by Jean-René Lacour.

Nicolas Flores

nicolas.flores@decathlon.com

1 Decathlon SportsLab, Movement Sciences Department, Villeneuve d'Ascq, France

2 Aix Marseille Univ, CNRS, ISM, Marseille, France

\section{Introduction}

Running is a performance activity, where running as long and/or as fast as possible is required to cover a given distance in a minimum of time. From a broad point of view, running performance is not limited to the small class of elite runners that are close to break the 120 min marathon barrier (Hoogkamer et al. 2017b; Sousa et al. 2018), but also includes non-elite and recreational runners that represent the greatest proportion of runners taking part in marathon races with finish times greater than $180 \mathrm{~min}$ (Deaner et al. 2015). A common physiological variable used to study the running performance is the running economy (RE). Many studies have investigated how some running shoe features improve RE, to decrease the metabolic energetic cost to run at a specific velocity or to run faster with the same metabolic energetic cost.

The decrease of shoe weight (Franz et al. 2012; Fuller et al. 2015; Hoogkamer et al. 2016), the increase of midsole material energy return (Frederick et al. 1986; Worobets et al. 2014; Sinclair et al. 2016), and the increase of shoe 
longitudinal bending stiffness (Roy and Stefanyshyn 2006) have been shown to improve the RE. These previous studies in the field of footwear have led Hoogkamer et al. (2017b) to propose that the combination of weight saving, high midsole material energy return, and high longitudinal bending stiffness in a same pair of shoes was potentially beneficial to improve the RE. A recent study has been the first to test this assumption by combining high energy return midsole material with high longitudinal bending stiffness in a same pair of shoes and has reported an improved RE over the average group of participants compared to baseline marathon racing shoes (Hoogkamer et al. 2017a). However, because the compared shoes conditions came from different brands, they varied in other features such as the midsole stiffness in compression, the midsole geometry, and upper shoe characteristics that may also influence the previously reported RE improvements. Therefore, it is not clear whether the improved RE was due to the combination of high energy return and high longitudinal bending stiffness features because other shoe features were not taken into account.

Understanding biomechanical responses induced by the interaction between the shoe and the body are crucial to better know how shoe features may influence the RE. It has been shown that shoes reported to improved RE, which combined high energy return midsoles and increased longitudinal bending stiffness, induced greater peak vertical ground reaction forces (GRF), longer ground contact times, and lower step frequencies (Hoogkamer et al. 2017a). Besides, decreased vertical GRF impulses (Heise and Martin 2001), increased vertical and leg stiffness (Butler et al. 2003), and a more aligned resultant GRF with the leg (Moore et al. 2016) have been shown to be beneficial for the RE. Analyzing the biomechanical responses induced by the midsole material energy return and/or the longitudinal bending stiffness in shoes with identical geometry and upper characteristics may help to understand how these shoe features can alter the metabolic energetic cost during running.

This study aimed to assess the effects of the midsole material energy return and the shoe longitudinal bending stiffness on the metabolic energetic cost of running and biomechanical parameters previously correlated to running economy improvements during overground running. A high energy return midsole material and/or high longitudinal bending stiffness was expected to decrease the energetic cost of running compared to shoes with lower energy return midsole material and/or lower longitudinal bending stiffness. These shoe features and their interaction would induce biomechanical results previously associated with RE improvements (Moore 2016; Hoogkamer et al. 2017a), that is: high energy return midsole material and high longitudinal bending stiffness would decrease GRF impulses, increase vertical and leg stiffness, increase ground contact time, decrease stride frequencies, and increase alignment between the resultant GRF and the leg during the stance phase.

\section{Methods}

\section{Participants}

Nineteen male recreational runners $(24 \pm 6$ years, $179.1 \pm 4.1 \mathrm{~cm}, 70.9 \pm 4.1 \mathrm{~kg}$, \#43 EU size), free from injury in the last year, took part in the experiment. All participants provided their written informed consent before participating in the study. The study protocol complied with the standards established in the Helsinki declarations and all the procedures have been approved by the Aix-Marseille university ethics committee.

\section{Design of shoe prototypes}

From the combination of low or high energy return and low or high longitudinal bending stiffness factors, four shoe prototype conditions were used in the study, and were as follows: low energy return midsole material and low longitudinal bending stiffness, high energy return midsole material and low longitudinal bending stiffness, low energy return midsole material and high longitudinal bending stiffness, high energy return midsole material and high longitudinal bending stiffness. The shoes had strictly identical appearance, upper material and geometry. The shoe mass was controlled by adding small masses on laces. Ten pairs per shoe condition ( 4 conditions $\times 10$ pairs) were used to limit the effects of material fatigue during the experiment. Thus, each shoe pair was worn by two participants maximum.

A conventional full ethylene-vinyl acetate foam (hardness 63 askerC) was used in the low energy return midsoles, and a higher level of energy return was obtained using full polyurethane foam (hardness 60 askerC) in the high energy return midsoles. The energy return refers to the midsole mechanical feature obtained from the area under the unloading curve of the force-displacement hysteresis during an impact drop test (American Society for Testing and Materials 2013). The impact drop test mechanical characterization performed on the rearfoot revealed that the high energy return condition was on average $29 \%$ greater than the low energy return condition (Table 1). To focus only on the effects of midsole energy return, the present study aimed to reduce as much as possible the difference in midsole material stiffness in compression between the low energy return condition and the high energy return conditions (difference of $16 \%$ on average). Compared to the maximum difference in stiffness in compression (176\%) swept by the running shoes of the footwear market (Shorten and Mientjes 2011), the difference in 
Table 1 Mean (SD) of shoe mechanical properties and shoe mass for the four shoe conditions combining low and high levels of energy return and longitudinal bending stiffness

\begin{tabular}{|c|c|c|c|c|}
\hline & $\begin{array}{l}\text { Low energy return/low } \\
\text { longitudinal bending } \\
\text { stiffness }\end{array}$ & $\begin{array}{l}\text { High energy return/low } \\
\text { longitudinal bending stiff- } \\
\text { ness }\end{array}$ & $\begin{array}{l}\text { Low energy return/high } \\
\text { longitudinal bending stiff- } \\
\text { ness }\end{array}$ & $\begin{array}{l}\text { High energy return/high } \\
\text { longitudinal bending } \\
\text { stiffness }\end{array}$ \\
\hline Energy return at rearpart (\%) & $48.2(0.6)$ & $62.2(1.3)$ & $48.2(0.6)$ & $62.3(1.6)$ \\
\hline $\begin{array}{l}\text { Local stiffness at rearpart } \\
\left(\mathrm{N} \mathrm{mm}^{-1}\right)\end{array}$ & $134.1(4.5)$ & $117.5(5.0)$ & $135.5(6.0)$ & $116.7(6.0)$ \\
\hline $\begin{array}{l}\text { Global stiffness at rearpart } \\
\left(\mathrm{N} \mathrm{mm}^{-1}\right)\end{array}$ & $82.1(1.8)$ & $68.3(2.7)$ & $81.6(2.6)$ & $68.0(2.3)$ \\
\hline $\begin{array}{l}\text { Longitudinal bending stiff- } \\
\text { ness at forepart }\left(\mathrm{N} \mathrm{mm}^{-1}\right)\end{array}$ & $19.2(1.0)$ & $15.4(1.0)$ & $43.4(2.0)$ & $38.0(1.8)$ \\
\hline Mass (g) & $367.7(2.9)$ & $368.9(1.3)$ & $367.2(1.1)$ & $369.6(2.1)$ \\
\hline
\end{tabular}

The energy return (area under the unloading curve), the local stiffness (linear slope of the loading curve between 95\% of the maximum force and this force minus $150 \mathrm{~N}$ ), and the global stiffness (linear slope of the loading curve between $10 \mathrm{~N}$ and the maximum force) were computed from the force-displacement curve of the impact drop test at the rearpart of shoes. The longitudinal bending stiffness (linear slope of the loading curve between 5 and $6 \mathrm{~mm}$ ) was computed from the force-displacement curve of the three-point bending test at the forepart of shoes

stiffness in compression swept by the shoe conditions was low in the present study.

The longitudinal bending stiffness was measured from a forefoot three-point bending test (Willwacher et al. 2013). The shoes were placed on two supporting points $80 \mathrm{~mm}$ apart from each other. A testing machine (E3000 ElectroPuls, Instron, Massachusetts, USA) applied $7.5 \mathrm{~mm}$ displacement in the shoe forefoot at $15 \mathrm{~mm} \mathrm{~s}^{-1}$. The required force to displace the stamp from 5 to $6 \mathrm{~mm}$ was considered as representative of the longitudinal bending stiffness. The higher level of longitudinal bending stiffness was obtained by adding flat carbon fiber composite plates of $0.9 \mathrm{~mm}$ thickness under the shoe insoles. The carbon plates were cut according to the insole shape and extended over the $2 / 3$ forepart of the shoe to avoid any combination with the midsole energy return factor at the rearfoot, assuming that participants landed on the ground with the heel first. The threepoint bending test showed that the high longitudinal bending stiffness condition was on average $135 \%$ greater than the low longitudinal bending stiffness condition (Table 1).

\section{Experimental protocol}

The participants performed three distinct running sessions with at least $48 \mathrm{~h}$ rest between each one. The sessions were: an outdoor incremental running test, an outdoor submaximal running test with physiological measurements, and an indoor submaximal running test with biomechanical measurements. The incremental test was always performed first, and both submaximal running tests were performed in a random order. All sessions were performed at the same day period to avoid any circadian rhythm effects (Atkinson and Reilly 1996), and overground to be as close as possible to an ecological and a realistic running session (Saunders et al. 2004; Chambon et al. 2015; Barnes and Kilding 2015).
The outdoor incremental running test was performed on a $250 \mathrm{~m}$ asphalt track. A 5 min warm-up at $7 \mathrm{~km} \mathrm{~h}^{-1}$ was done before increasing the speed by $1 \mathrm{~km} \mathrm{~h}^{-1}$ every 2 min while participants wore their own running shoes. The speed was imposed with ground marks and a metronome. A wearable expired gas analysis by indirect calorimetry (K5, Cosmed, Italy) recorded breath-by-breath data. The standard error of measurement of this device was reported to be $1.6 \%$ for the rate of oxygen uptake and $2.2 \%$ for the rate of carbon dioxide production (Baldari et al. 2015). The device was calibrated before each participant with a reference gas containing known concentrations of oxygen and carbon dioxide, and with a $3.0 \mathrm{~L}$ syringe to ensure an accurate volume measurement. The test was performed until participants reached their ventilatory anaerobic threshold (VAT), determined as described elsewhere (Mezzani et al. 2009). The speed corresponding to the VAT was kept for each participant. The speed achieved during the experiment $\left(10.8 \pm 1.1 \mathrm{~km} \mathrm{~h}^{-1}\right.$ on average) was equal to $90 \%$ of the speed that elicited VAT, and corresponded to marathon finish time between 180 and $210 \mathrm{~min}$ in recreational runners (Gordon et al. 2017).

The outdoor submaximal running test was performed on a $250 \mathrm{~m}$ asphalt track. The four shoe conditions were presented to the participants in a random order. After a $5 \mathrm{~min}$ warm-up at $90 \%$ of their VAT with their own shoes, the participants ran with each shoe condition during $8 \mathrm{~min}$ at $90 \%$ of their VAT (imposed speed with ground marks and a metronome) to ensure being below the anaerobic threshold during the whole experiment. A 5 min rest was kept between each shoe condition. Breath-by-breath data were recorded during the entire test. As described above, the wearable expired gas analyzer was calibrated before each participant. An accelerometer (wGT3X-BT, Actigraph, Florida, sampling rate $100 \mathrm{~Hz}$ ) firmly strapped on the posterior aspect 
of the sacrum was used to compute the number of strides performed during a given time (i.e., stride frequency).

The indoor submaximal running test was performed in a $50 \mathrm{~m}$ laboratory. The participants ran continuously on the indoor concrete track to be in accordance with the outdoor submaximal running test as much as possible. After a $5 \mathrm{~min}$ warm-up at $90 \%$ of their VAT with their own shoes, the participants ran with each shoe condition at $90 \%$ of their VAT (imposed speed with ground marks and a metronome), while five valid trials were kept from the 6th min of running. Six minutes adaptation enabled to reach a physiological steady state (Barnes and Kilding 2015), a stable running pattern (Delattre et al. 2013), and stable shoe mechanical properties (Divert et al. 2005). A trial was valid when the participants kept their natural running pattern before landing with the right foot fully on the force plate (9287CA, Kistler, Switzerland). The GRF data was acquired at $2000 \mathrm{~Hz}$. The tridimensional coordinates of fifty-eight retro-reflective markers placed on the full body were recorded with a ten-camera optical motion capture system at $200 \mathrm{~Hz}$ (Oqus 7 Camera series, Qualisys, Sweden).

\section{Data analysis}

Gas exchange data were not filtered and the RE was expressed as energetic cost in $\mathrm{kJ} \mathrm{kg}^{-1} \mathrm{~km}^{-1}$ (Fletcher et al. 2009). The caloric equivalent conversions based on the Weir's equation (1949) were obtained from the expired gas analysis by indirect calorimetry device. The RE, the oxygen uptake, the carbon dioxide production, and the respiratory exchange ratio were averaged from 6 to $7 \mathrm{~min} 30 \mathrm{~s}$. This time period corresponded to steady-state conditions checked by a respiratory exchange ratio lower than 1 and an increase of $<100 \mathrm{ml} \mathrm{O}_{2}$ (Saunders et al. 2004; Barnes and Kilding 2015).

Adapted from a previous method (Neville et al. 2010), the accelerometer data were low-pass filtered with a secondorder critically damped filter with a $2 \mathrm{~Hz}$ cutoff frequency to obtain a sine form signal. Each extremum of this signal represented a ground contact. The number of ground contacts (n) from 6 to $7 \mathrm{~min} 30 \mathrm{~s}$ and the corresponding elapsed time $(t)$ were used to determine the stride frequency $(f=n / t)$.

The GRF data were low-pass filtered with a second-order critically damped filter with a $40 \mathrm{~Hz}$ cutoff frequency. The ground stance phase was identified as vertical GRF greater than $10 \mathrm{~N}$. The net vertical impulse, the braking impulse, and the propulsion impulse normalized to the body weight (BW) were computed as previously described (Heise and Martin 2001). The anteroposterior and vertical GRF waveforms were time normalized to the stance duration (101 points) and displayed in two separate matrices (380 rows [19 participants $\times 4$ shoe conditions $\times 5$ trials] and 101 columns [percentage of stance phase]) for the statistical analysis on the whole curve (Statistical Parametric Mapping, more details below in the "Statistical analysis" section).

The coordinates of retro-reflective markers were lowpass filtered with a second-order Butterworth filter with a $10 \mathrm{~Hz}$ cutoff frequency. The body center of mass position was computed as the barycenter of all segmental center of mass during the stance phase from Visual3D (v6 Professional, C-Motion, USA). The segment inertia parameters used to compute the body center of mass were obtained from de Leva (1996). The maximum vertical displacement of the center of mass and the corresponding vertical force were used to compute the vertical stiffness (Butler et al. 2003). The leg stiffness was calculated from the horizontal velocity of the center of mass, the ground contact time, the standing leg length, the maximum vertical displacement of the center of mass, and the corresponding vertical force (Butler et al. 2003). Then, adapted from Moore et al. (2016), a leg axis was defined as the vector between the lateral malleolus marker and the center of mass. The angle between the leg axis and the vertical axis defined the leg angle. The resultant GRF angle was also determined according to the vertical axis. The alignment of the leg axis with the resultant GRF was finally computed in the sagittal plane as the difference between the leg angle and the resultant GRF angle during the whole stance phase and included for the statistical analysis on the whole curve.

\section{Statistical analysis}

The normality of residuals and the homogeneity of variance were checked before performing two-way repeated measures analysis of variance (ANOVA) to evaluate $(\alpha=0.05)$ the main effects of the factors "energy return" and "longitudinal bending stiffness" and the interaction of these factors on parameters expressed as scalar values (i.e., running economy, ground contact time, etc.). When the normality of residuals or homogeneity of variances was not met, a permutation procedure was performed. To complete, the partial omega squared $\left(\omega_{P}^{2}\right)$, appropriate to the present study design (Lakens 2013), was used to determine small (0.02), medium $(0.13)$ and large (0.26) effect sizes.

The statistical parametric mapping (SPM) procedure (Pataky et al. 2013, 2015) was used to determine the potential statistical differences between shoe features on biomechanical variables over the whole stance phase duration. The use of SPM is appropriate if the hypothesis is "non-directed" (Pataky et al. 2013), meaning that an effect of shoe features over the whole stance phase was expected, rather than at specific scalar time events (e.g., GRF peaks representing discrete events of only $1 \%$ or less of the ground contact). This was the case for the hypotheses on the GRF and the alignment of the resultant GRF with the leg waveforms in the present study. The SPM principle was identical to the classical 
univariate statistical procedure on scalar values except that the test statistic field (i.e., the $F$ value as a function of time noted SPM $\{F\}$ in the present study) was computed for each of the scalar points of the waveforms. Due to the repeated test statistic over time, the critical test statistic threshold and the $p$ value were computed and corrected through the entire temporal domain by considering the data smoothness, the data size, and the random field behavior theory to retain a family-wise Type I error rate $\alpha=0.05$ (Pataky et al. 2013). When the test statistic field exceeded the critical test statistic threshold, a significant effect was observed at the corresponding time period. The greater the test statistic field exceeds the critical test statistic threshold, the more the effect was significant. The SPM was performed from the Matlab open-source spm1d code (M.0.4.3, http://www. spm1d.org). Two-way repeated measures ANOVA evaluated $(\alpha=0.05)$ the main effects of the factors "energy return" and "longitudinal bending stiffness" and the interaction between these factors on the GRF waveforms and the alignment of the resultant GRF with the leg waveform.

\section{Results}

\section{Shoe midsole energy return}

The energy return shoe feature did not alter $(p>0.05)$ the energetic cost of running (Table 2).

The high energy return shoes induced a significantly lower stride frequency $\left(p=0.024, \omega_{P}^{2}=0.20\right.$, medium), a longer contact time $\left(p=0.017, \omega_{P}^{2}=0.23\right.$, large $)$, and a longer propulsion time $\left(p=0.005, \omega_{P}^{2}=0.31\right.$, large $)$ compared to the low energy return shoes.

SPM analysis revealed that the high energy return shoes induced more braking anteroposterior GRF from 23 to $25 \%\left(F_{1,18}>13.60 ; p=0.029\right)$, and more vertical

Table 2 Mean (SD) of physiological and biomechanical parameters for the four shoe conditions combining low and high levels of energy return and longitudinal bending stiffness

\begin{tabular}{|c|c|c|c|c|c|c|c|}
\hline & $\begin{array}{l}\text { Low energy } \\
\text { return/low lon- } \\
\text { gitudinal bend- } \\
\text { ing stiffness }\end{array}$ & $\begin{array}{l}\text { High energy } \\
\text { return/low lon- } \\
\text { gitudinal bend- } \\
\text { ing stiffness }\end{array}$ & $\begin{array}{l}\text { Low energy } \\
\text { return/high lon- } \\
\text { gitudinal bend- } \\
\text { ing stiffness }\end{array}$ & $\begin{array}{l}\text { High energy } \\
\text { return/high lon- } \\
\text { gitudinal bend- } \\
\text { ing stiffness }\end{array}$ & $\begin{array}{l}\text { Main effect } \\
\text { energy return }\end{array}$ & $\begin{array}{l}\text { Main effect } \\
\text { longitudinal } \\
\text { bending stiff- } \\
\text { ness }\end{array}$ & Interaction effect \\
\hline $\begin{array}{l}\mathrm{RE} \\
\left(\mathrm{kJ} \mathrm{kg}^{-1} \mathrm{~km}^{-1}\right)\end{array}$ & $4.75(0.50)$ & $4.73(0.51)$ & $4.72(0.49)$ & $4.76(0.51)$ & $\begin{array}{l}F=0.22 \\
p=0.630\end{array}$ & $\begin{array}{l}F=0.05 \\
p=0.860\end{array}$ & $\begin{array}{l}\mathrm{F}=1.06 \\
p=0.350\end{array}$ \\
\hline $\mathrm{VO}_{2}\left(1 \mathrm{~min}^{-1}\right)$ & $2.98(0.32)$ & $2.97(0.36)$ & $2.95(0.34)$ & $2.98(0.34)$ & $\begin{array}{r}\mathrm{Fn}=0.76 ; \\
p=0.388\end{array}$ & $\begin{aligned} \mathrm{Fn} & =0.32 ; \\
p & =0.588\end{aligned}$ & $\begin{aligned} \mathrm{Fn} & =0.92 ; \\
p & =0.338\end{aligned}$ \\
\hline $\mathrm{VCO}_{2}\left(1 \mathrm{~min}^{-1}\right)$ & $2.62(0.28)$ & $2.65(0.28)$ & $2.61(0.29)$ & $2.64(0.29)$ & $\begin{array}{c}F=3.16 \\
p=0.090\end{array}$ & $\begin{array}{l}\mathrm{F}=0.22 \\
p=0.621\end{array}$ & $\begin{array}{l}\mathrm{F}=0.01 ; \\
p=0.925\end{array}$ \\
\hline $\begin{array}{l}\text { Respiratory } \\
\text { exchange ratio }\end{array}$ & $0.88(0.07)$ & $0.89(0.07)$ & $0.89(0.07)$ & $0.89(0.07)$ & $\begin{aligned} \mathrm{Fn} & =4.12 ; \\
p & =0.057\end{aligned}$ & $\begin{aligned} \mathrm{Fn} & =0.01 ; \\
p & =0.931\end{aligned}$ & $\begin{aligned} \mathrm{Fn} & =0.71 ; \\
p & =0.404\end{aligned}$ \\
\hline $\begin{array}{l}\text { Stride frequency } \\
(\mathrm{Hz})^{\mathrm{EnR}}\end{array}$ & $1.34(0.05)$ & $1.33(0.05)$ & $1.34(0.04)$ & $1.33(0.05)$ & $\begin{array}{l}\mathrm{F}=5.95 \\
p=0.024\end{array}$ & $\begin{aligned} \mathrm{F} & =0.37 \\
p & =0.550\end{aligned}$ & $\begin{array}{l}\mathrm{F}=1.12 ; \\
p=0.303\end{array}$ \\
\hline $\begin{array}{l}\text { Contact time } \\
(\mathrm{ms})^{\mathrm{EnR}, \mathrm{BS}}\end{array}$ & $271.9(17.7)$ & $275.6(18.6)$ & $277.0(18.7)$ & $278.7(18.8)$ & $\begin{array}{l}\mathrm{F}=6.96 \\
p=0.017\end{array}$ & $\begin{array}{c}\mathrm{F}=10.02 \\
p=0.005\end{array}$ & $\begin{array}{l}\mathrm{F}=0.68 \\
\quad p=0.421\end{array}$ \\
\hline $\begin{array}{l}\text { Braking time } \\
\quad(\mathrm{ms})\end{array}$ & $133.0(13.0)$ & $133.4(13.4)$ & 133.4 (14.4) & $132.9(14.0)$ & $\begin{array}{c}\mathrm{Fn}<0.01 \\
\quad p=0.994\end{array}$ & $\begin{array}{c}\mathrm{Fn}<0.01 \\
\quad p=0.983\end{array}$ & $\begin{aligned} \mathrm{Fn} & =0.19 ; \\
p & =0.983\end{aligned}$ \\
\hline $\begin{array}{l}\text { Propulsion time } \\
(\mathrm{ms})^{\text {EnR, BS }}\end{array}$ & $139.0(10.7)$ & $142.2(11.7)$ & $143.7(12.0)$ & $145.7(11.1)$ & $\begin{aligned} \mathrm{Fn} & =10.17 \\
p & =0.005\end{aligned}$ & $\begin{array}{r}\mathrm{Fn}=24.42 \\
p<0.001\end{array}$ & $\begin{aligned} \mathrm{Fn} & =0.20 \\
p & =0.661\end{aligned}$ \\
\hline $\begin{array}{l}\text { Net verti- } \\
\text { cal impulse } \\
(\mathrm{BW} \mathrm{ms})^{\mathrm{BS}}\end{array}$ & $244.9(16.5)$ & 245.8 (17.9) & $241.2(17.2)$ & $242.1(17.8)$ & $\begin{aligned} \mathrm{Fn} & =0.44 \\
p & =0.523\end{aligned}$ & $\begin{aligned} \mathrm{Fn} & =5.91 ; \\
p & =0.023\end{aligned}$ & $\begin{aligned} \mathrm{Fn} & <0.01 \\
p & =0.985\end{aligned}$ \\
\hline $\begin{array}{l}\text { Braking impulse } \\
\text { (BW ms) }\end{array}$ & $-22.5(4.4)$ & $-22.6(4.3)$ & $-22.6(5.8)$ & $-22.4(4.4)$ & $\begin{aligned} \mathrm{Fn} & =0.03 ; \\
p & =0.862\end{aligned}$ & $\begin{aligned} \mathrm{Fn} & =0.01 ; \\
p & =0.912\end{aligned}$ & $\begin{aligned} \mathrm{Fn} & =0.05 ; \\
p & =0.822\end{aligned}$ \\
\hline $\begin{array}{l}\text { Propulsion } \\
\text { impulse } \\
\text { (BW ms) }\end{array}$ & $22.3(3.8)$ & $22.4(3.6)$ & $21.8(4.3)$ & $22.1(3.7)$ & $\begin{aligned} \mathrm{Fn} & =0.64 \\
p & =0.438\end{aligned}$ & $\begin{aligned} \mathrm{Fn} & =3.21 \\
p & =0.083\end{aligned}$ & $\begin{aligned} \mathrm{Fn} & =0.06 \\
p & =0.814\end{aligned}$ \\
\hline $\begin{array}{l}\text { Vertical stiffness } \\
\left(\mathrm{kN} \mathrm{m}^{-1}\right)^{\mathrm{BS}}\end{array}$ & $23.2(3.9)$ & $23.2(2.9)$ & $24.0(2.9)$ & $23.7(3.0)$ & $\begin{aligned} \mathrm{Fn} & =0.67 \\
p & =0.420\end{aligned}$ & $\begin{array}{c}\mathrm{Fn}=5.59 \\
p 0.032\end{array}$ & $\begin{aligned} \mathrm{Fn} & =0.17 \\
p & =0.686\end{aligned}$ \\
\hline $\begin{array}{l}\text { Leg stiffness } \\
\left(\mathrm{kN} \mathrm{m}^{-1}\right)\end{array}$ & $10.9(2.0)$ & $10.9(1.6)$ & $10.9(1.5)$ & $10.8(1.6)$ & $\begin{aligned} \mathrm{Fn} & =0.07 ; \\
p & =0.789\end{aligned}$ & $\begin{aligned} \mathrm{Fn} & =0.07 \\
p & =0.800\end{aligned}$ & $\begin{aligned} \mathrm{Fn} & =0.07 \\
p & =0.786\end{aligned}$ \\
\hline
\end{tabular}

$\mathrm{EnR}$ and ${ }^{\mathrm{BS}}$ indicated significant differences $(p<0.05)$ between low and high levels of energy return or longitudinal bending stiffness, respectively. $\mathrm{F}$ and Fn were the test statistic values from the parametric and non-parametric procedures, respectively 
GRF from 15 to $18 \%$ of the stance phase $\left(F_{1,18}>13.88\right.$; $p=0.013)$ compared to the low energy return shoes (Figs. 1, 2). From 1 to $4 \%$ of the stance phase, the high energy return shoes induced less vertical GRF $\left(F_{1,18}>13.88 ; p=0.019\right)$.

The high energy return shoes induced a lower alignment of the resultant GRF relative to the leg from 23 to $25 \%\left(F_{1,18}>13.91 ; p=0.018\right)$ and a greater alignment from 69 to $72 \%$ of the stance phase $\left(F_{1,18}>13.91\right.$; $p=0.010)$ compared to the low energy return shoes (Fig. 3).

\section{Shoe longitudinal bending stiffness}

The longitudinal bending stiffness shoe feature did not alter $(p>0.05)$ the energetic cost of running (Table 2).

The high longitudinal bending stiffness shoes induced a significantly longer contact time $\left(p=0.005, \omega_{P}^{2}=0.31\right.$, large), a longer propulsion time $\left(p<0.001, \omega_{P}^{2}=0.54\right.$, large), a greater vertical stiffness $\left(p=0.032, \omega_{P}^{2}=0.19\right.$, medium $)$, and a lower net vertical impulse $\left(p=0.023, \omega_{P}^{2}=0.20\right.$, medium) compared to the low longitudinal bending stiffness shoes.

The high longitudinal bending stiffness shoes induced less propulsive anteroposterior GRF from 71 to $88 \%$
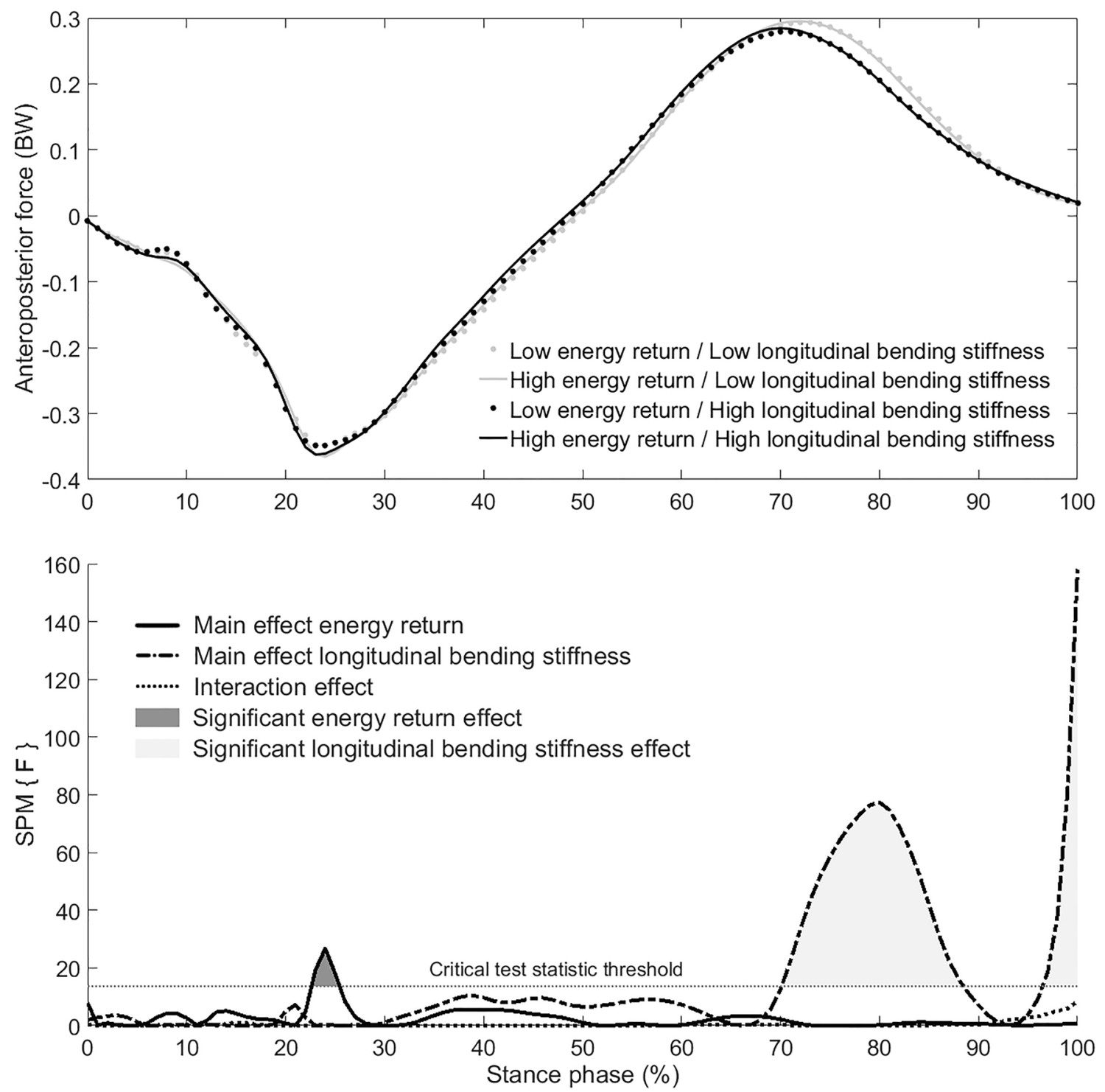

Fig. 1 (Top) Mean anteroposterior ground reaction force time- and weight-normalized. Negative and positive values were braking and propulsion forces, respectively. Standard deviations were not pre- sented for more clarity. (Down) SPM $\{F\}$ curves of the main effects energy return, longitudinal bending stiffness, and their interaction 

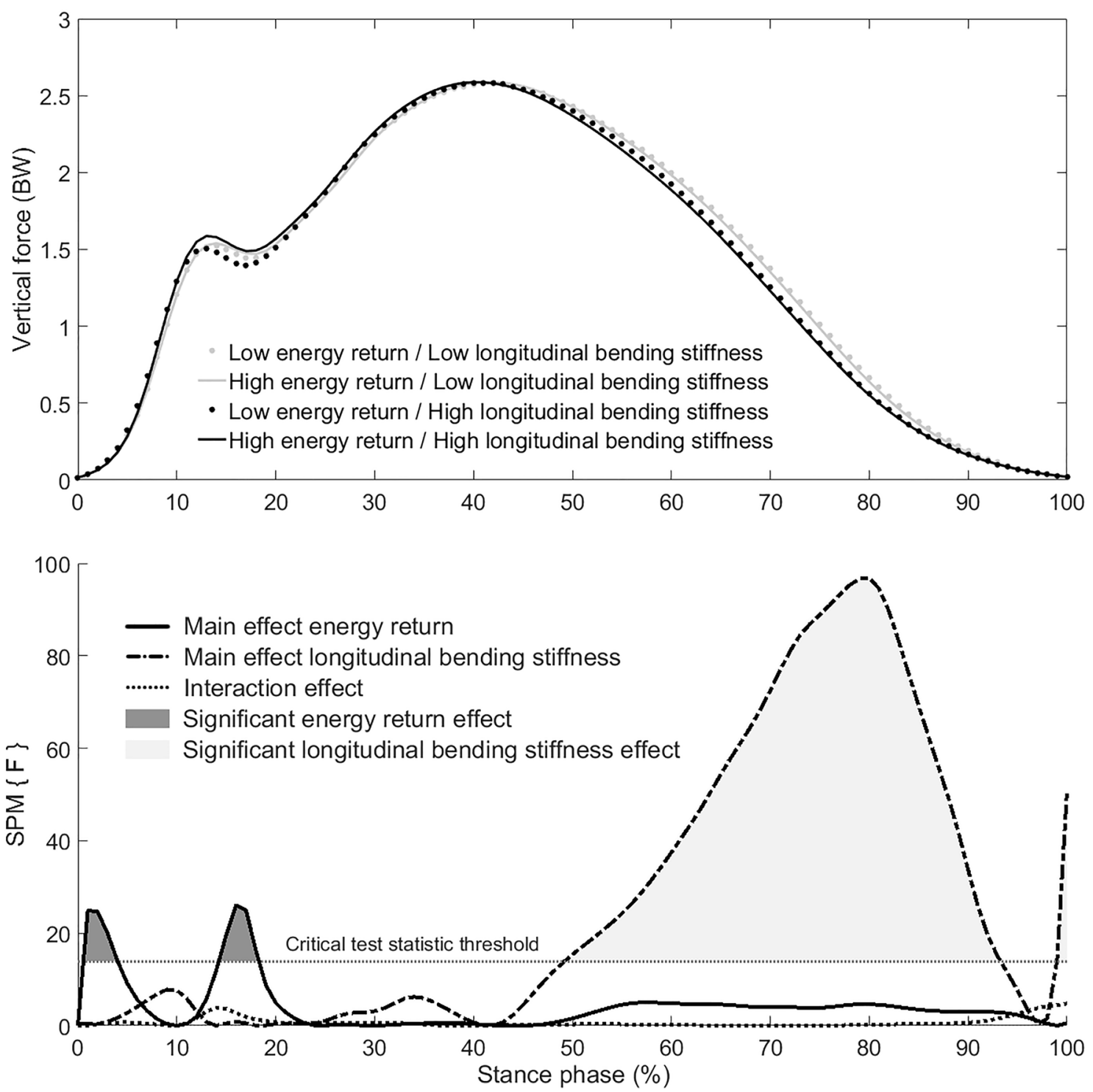

Fig. 2 (Top) Mean vertical ground reaction force time- and weight-normalized. Standard deviations were not presented for more clarity. (Down) SPM $\{F\}$ curves of the main effects energy return, longitudinal bending stiffness, and their interaction

$\left(F_{1,18}>13.60 ; p<0.001\right)$, and less vertical GRF from 50 to $93 \%$ of the stance phase $\left(F_{1,18}>13.88 ; p<0.001\right)$ compared to the low longitudinal bending stiffness shoes (Figs. 1, 2). The high longitudinal bending stiffness shoes induced more anteroposterior GRF and vertical GRF from 97 to $100 \%\left(F_{1,18}>13.60 ; p=0.022\right)$ and from 99 to $100 \%$ of the stance phase $\left(F_{1,18}>13.88 ; p=0.046\right)$, respectively.

The high longitudinal bending stiffness shoes induced a lower alignment around $20 \%\left(F_{1,18}>13.91 ; p=0.048\right)$, $49 \%\left(F_{1,18}>13.91 ; p=0.045\right)$ and from 95 to $100 \%$ of the stance phase $\left(F_{1,18}>13.91 ; p=0.006\right)$ compared to the low longitudinal bending stiffness shoes (Fig. 3).

\section{Interaction between shoe midsole energy return and shoe longitudinal bending stiffness}

No interaction effect of the two shoe features was observed on all the parameters analyzed in the present study $(p>0.05)$.

\section{Discussion}

This study assessed the effects of shoe energy return and shoe longitudinal bending stiffness, while controlling for other shoe features, on the energetic cost of transport during 

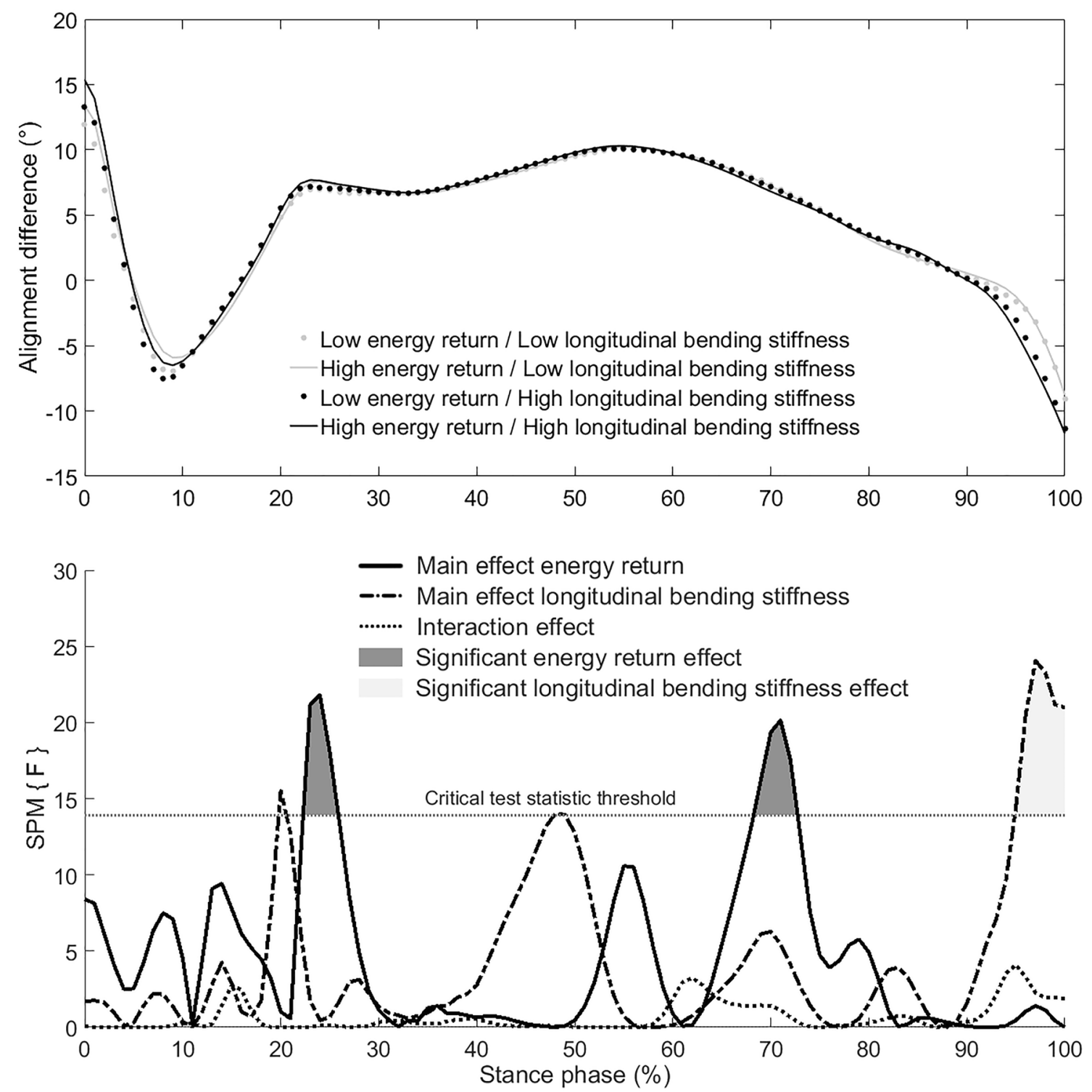

Fig. 3 (Top) Mean alignment between the resultant ground reaction force and the leg time-normalized. Negative and positive values indicated a more forward and a more backward orientation of the resultant ground reaction force compared to the leg, respectively. The

overground running on hard surfaces, with a special focus on biomechanical parameters related to running economy.

\section{Shoe midsole energy return}

The 29\% difference in terms of energy return between the shoe conditions in the present study did not induce any effect on the metabolic energetic cost of running. A greater energy return was expected to induce RE improvements as shoe conditions that induced RE improvements in previous studies had 7\% more energy return (Frederick et al. 1986), $15 \%$ more energy return (Worobets et al. 2014), and from closer the values were to zero, the better the alignment between the resultant GRF and the leg. Standard deviations were not presented for more clarity. (Down) SPM $\{F\}$ curves of the main effects energy return, longitudinal bending stiffness, and their interaction

15 to $33 \%$ more energy return (Hoogkamer et al. 2017a) compared to their respective control conditions. It should be highlighted that the present study has reduced as much as possible the difference in midsole stiffness in compression $(16 \%)$ between low and high energy return shoe conditions. This $16 \%$ difference was substantially lower than the $30 \%$ and $50 \%$ differences, respectively, measured between shoe conditions in the studies of Worobets et al. (2014) and Hoogkamer et al. (2017a, computed from available force and displacement data), while no data concerning the stiffness in compression are available from Frederick et al. (1986). Consequently, one can suppose that combining low midsole 
stiffness in compression and high midsole energy return (as in previous studies), and not only the midsole energy return (as in the present study), is beneficial to improve RE. More widely, the potential beneficial shoe feature combination to reduce the energetic cost of running, that is weight saving, high midsole material energy return, and high longitudinal bending stiffness in a same pair of shoes (Hoogkamer et al. 2017b), should be completed with low midsole material stiffness in compression.

Although the energetic cost of running was not significantly altered, the high energy return shoes induced lower stride frequency, longer contact time, and higher vertical GRF after the first peak. This was well in line with a previous study showing that these biomechanical changes induced by high energy return shoes did not correlate with RE improvements (Hoogkamer et al. 2017a). Furthermore, although the high energy return shoes induced slightly more alignment between the resultant GRF and the leg around the propulsive peak time period, this alignment $\left(6.6^{\circ}\right)$ remained substantially far from the previously reported almost perfect alignment during the propulsive peak time period that can improve RE (1.6 ${ }^{\circ}$, Moore et al. 2016). This may explain why the RE was not significantly altered in the present study.

\section{Shoe longitudinal bending stiffness}

The energetic cost of overground running was not influenced by the shoe longitudinal bending stiffness although the high longitudinal bending stiffness conditions $\left(40.7 \pm 3.3 \mathrm{~N} \mathrm{~mm}^{-1}\right)$ were quite close to the intermediate longitudinal bending stiffness that induced RE improvements in the study of Roy and Stefanyshyn $\left(38 \mathrm{~N} \mathrm{~mm}^{-1}\right.$, 2006). These different findings may be due to the slower speed achieved by the recreational runners in the present study $\left(10.8 \pm 1.1 \mathrm{~km} \mathrm{~h}^{-1}\right)$ compared to the speed achieved in the study of Roy and Stefanyshyn $\left(13.3 \mathrm{~km} \mathrm{~h}^{-1}, 2006\right)$, which may have altered the shoe mechanical loading in flexion and potentiated the effects of longitudinal bending stiffness on RE. Furthermore, Oh and Park (2017) have shown that the increase in longitudinal bending stiffness should be dependent on the stiffness of the runner's metatarsophalangeal joint to improve the RE. Although the metatarsophalangeal joint stiffness was not measured in the present study and based on the absence of RE alteration, one can suppose that the longitudinal bending stiffness used in the present study $\left(40.7 \pm 3.3 \mathrm{~N} \mathrm{~mm}^{-1}\right)$ was not suitable for all participants' forefoot characteristics. Thus, care should be taken in recruiting participants when the longitudinal bending stiffness shoe feature is investigated.

The high longitudinal bending stiffness shoes induced lower net vertical impulse and greater vertical stiffness that are habitually not associated with longer contact time to be potentially beneficial for RE (Moore 2016). Furthermore, the greater forward GRF observed just before toe-off supports previous thoughts stating that the pushing force is induced by the action of the stiff carbon plate recovering its initial shape in the shoe forefoot (Willwacher et al. 2014). However, the use of stiff plates induced a decreased alignment between the resultant GRF and the leg in the present study, which came along with no improvement of the energetic cost of running. Thus, it is likely that a higher pushing force induced by the carbon plate may improve the RE if this force is optimally oriented relative to the runner's center of mass.

\section{Interaction between shoe midsole energy return and shoe longitudinal bending stiffness}

The present study did not observe any specific effect of combined low or high midsole material energy return with low or high longitudinal bending stiffness that improved the energetic cost of overground running. This result is different from those of Hoogkamer et al. (2017a) although the comparisons are difficult due to some methodological differences. First, both studies did not exactly answer to the same purpose. Hoogkamer et al. (2017a) assessed the effect of three different shoes on RE, while the present study assessed the main effects and the interaction of shoe features of identical prototypes on RE. Second, the stiff plates used in the study of Hoogkamer et al. (2017a) were located at the bottom of the midsole under the forefoot area, while the stiff plates located under the insoles in the present study may have decreased the forefoot penetration into the midsole. Thus, the stiff plate located under insoles may have decreased the perceived comfort, which can in turn decreased RE (Luo et al. 2009). Finally, the sub-elite runners from Hoogkamer et al. (2017a) ran at substantially faster running speeds (14, 16 , and $\left.18 \mathrm{~km} \mathrm{~h}^{-1}\right)$ than the recreational runners of the present study $\left(10.8 \pm 1.1 \mathrm{~km} \mathrm{~h}^{-1}\right)$. It is likely that running speed may have altered the mechanical loading (compression and/ or flexion) of the shoes, and thus may have potentiated the effects of midsole energy return and/or longitudinal bending stiffness on RE. It may be a threshold speed above which the interactions of runner and shoe characteristics are optimized to induce positive effects on the energetic cost and biomechanics of running. Therefore, the present results might not be transportable to sub-elite and elite runners.

\section{Conclusion}

This study analyzed the effects of the energy return and the longitudinal bending stiffness shoe features on the energetic cost and biomechanical responses during running. Special attention was paid to control methodological points due to the shoe features (identical mass, geometry and upper characteristics, difference in midsole stiffness in compression 
was reduced as much as possible), the running surface (overground on hard surfaces), and the running speed (relative to the participants' physical capacity). In these conditions, neither the high energy return, nor the high longitudinal bending stiffness, nor the combinations between these shoe features altered the energetic cost of overground running in recreational runners. The high energy return and the high longitudinal bending stiffness shoe features induced subtle biomechanical adaptations previously associated with an improved RE, but probably not large enough to induce any acute effect on the energetic cost of running. However, it is still likely that these shoe features may positively affect the RE after a familiarization period or during prolonged running by preventing the increase of the energetic cost due to runners' fatigue. To potentially induce benefits on RE, footwear manufacturers should consider low stiffness in compression in addition to high energy return, rather than only high energy return in the midsoles of running shoes, while the longitudinal bending stiffness should be adapted to the runner's characteristics.

Acknowledgements Authors would thank Marvin Dufrenne, Delphine Chadefaux and Romain Hardouin (Aix-Marseille University) for their help during the data acquisition. Authors also thank Alexia Cariou and Estelle Le Gendre (Decathlon SportsLab) for their statistical support, and Dr. Todd Pataky (Kyoto University) for the suitable use of the Statistical Parametric Mapping procedure. Special thanks to Dr. Cédric Morio (Decathlon SportsLab) for his useful advices about the experimental procedure and the data analysis, and Mikku Knudsen (Decathlon SportsLab) for his English review.

Author contributions NF performed the research design, the experiments, the data analysis, and the manuscript writing. ND, EB and GR contributed in the research design and in the manuscript writing. ND and GR contributed in the data analysis. All authors read and approved the manuscript.

\section{Compliance with ethical standards}

Conflict of interest NF and ND are members of the company Decath$\operatorname{lon}^{\mathrm{TM}}$ in the research department. NF and ND did not report any conflict of interest because the shoe conditions used in this study were prototypes without any commercial value. NF, ND, EB and GR declared that the results of the study are presented clearly, honestly, and without fabrication, falsification, nor inappropriate data manipulation.

Ethical approval All procedures performed in this study involving human participants were in accordance with the ethical standards of the Aix-Marseille University institutional research and with the 1964 Helsinki declaration and its later amendments or comparable ethical standards.

\section{References}

American Society for Testing and Materials AF-13 (2013) Standard test method for impact attenuation of athletic shoe cushioning systems and materials
Atkinson G, Reilly T (1996) Circadian variation in sports performance. Sports Med 21:292-312

Baldari C, Meucci M, Bolletta F, Gallotta MC, Emerenziani GP, Guidetti L (2015) Accuracy and reliability of COSMED K5 portable metabolic device versus simulating system. Sport Sci Health 11:S58

Barnes KR, Kilding AE (2015) Running economy: measurement, norms, and determining factors. Sport Med Open 1:8. https:// doi.org/10.1186/s40798-015-0007-y

Butler RJ, Crowell HP, Davis IM (2003) Lower extremity stiffness: implications for performance and injury. Clin Biomech (Bristol Avon) 18:511-517

Chambon N, Delattre N, Guéguen N, Berton E, Rao G (2015) Shoe drop has opposite influence on running pattern when running overground or on a treadmill. Eur J Appl Physiol 115:911-918. https://doi.org/10.1007/s00421-014-3072-x

de Leva P (1996) Adjustments to Zatsiorsky-Seluyanov's segment inertia parameters. J Biomech 29:1223-1230

Deaner RO, Carter RE, Joyner MJ, Hunter SK (2015) Men are more likely than women to slow in the marathon. Med Sci Sport Exerc 47(3):607-616. https://doi.org/10.1249/MSS.0000000000 000432

Delattre N, Chambon N, Berton E, Gueguen N, Rao G (2013) Effect of time during a running session with minimal footwear. Comput Methods Biomech Biomed Eng 16:104-105. https://doi. org/10.1080/10255842.2013.815924

Divert C, Baur H, Mornieux G, Mayer F, Belli A (2005) Stiffness adaptations in shod running. J Appl Biomech 21:311-321

Fletcher JR, Esau SP, MacIntosh BR (2009) Economy of running: beyond the measurement of oxygen uptake. J Appl Physiol 107:1918-1922. https://doi.org/10.1152/japplphysiol.00307.2009

Franz JR, Wierzbinski CM, Kram R (2012) Metabolic cost of running barefoot versus shod. Med Sci Sport Exerc 44:1519-1525. https ://doi.org/10.1249/MSS.0b013e3182514a88

Frederick EC, Howley ET, Powers SK (1986) Lower oxygen demands of running in soft-soled shoes. Res Q Exerc Sport 57:174-177. https://doi.org/10.1080/02701367.1986.10762196

Fuller JT, Bellenger CR, Thewlis D, Tsiros MD, Buckley JD (2015) The effect of footwear on running performance and running economy in distance runners. Sport Med 45:411-422. https://doi. org/10.1007/s40279-014-0283-6

Gordon D, Wightman S, Basevitch I, Johnstone J, Espejo-Sanchez C, Beckford C, Boal M, Scruton A, Ferrandino M, Merzbach V (2017) Physiological and training characteristics of recreational marathon runners. Open Access J Sports Med 8:231-241. https ://doi.org/10.2147/OAJSM.S141657

Heise GD, Martin PE (2001) Are variations in running economy in humans associated with ground reaction force characteristics? Eur J Appl Physiol 84:438-442. https://doi.org/10.1007/s0042 10100394

Hoogkamer W, Kipp S, Spiering BA, Kram R (2016) Altered running economy directly translates to altered distance-running performance. Med Sci Sport Exerc 48:2175-2180. https://doi. org/10.1249/MSS.0000000000001012

Hoogkamer W, Kipp S, Frank JH, Farina EM, Luo G, Kram R (2017a) A comparison of the energetic cost of running in marathon racing shoes. Sport Med. https://doi.org/10.1007/s40279-017-0811-2

Hoogkamer W, Kram R, Arellano CJ (2017b) How biomechanical improvements in running economy could break the 2-hour marathon barrier. Sport Med. https://doi.org/10.1007/s4027 9-017-0708-0

Lakens D (2013) Calculating and reporting effect sizes to facilitate cumulative science: a practical primer for $t$ tests and ANOVAs. Front Psychol 4:1-12. https://doi.org/10.3389/fpsyg.2013.00863

Luo G, Stergiou P, Worobets J, Nigg B, Stefanyshyn D (2009) Improved footwear comfort reduces oxygen consumption during 
running. Footwear Sci 1:25-29. https://doi.org/10.1080/19424 280902993001

Mezzani A, Agostoni P, Cohen-Solal A, Corrà U, Jegier A, Kouidi E, Mazic S, Meurin P, Piepoli M, Simon A, Van Laethem C, Venhees L (2009) Standards for the use of cardiopulmonary exercise testing for the functional evaluation of cardiac patients: a report from the Exercise Physiology Section of the European Association for Cardiovascular Prevention and Rehabilitation. Eur J Cardiovasc Prev Rehabil 16:249-267. https://doi.org/10.1097/HJR.0b013 e32832914c8

Moore IS (2016) Is there an economical running technique? A review of modifiable biomechanical factors affecting running economy. Sport Med 46:793-807. https://doi.org/10.1007/s4027 9-016-0474-4

Moore IS, Jones AM, Dixon SJ (2016) Reduced oxygen cost of running is related to alignment of the resultant GRF and leg axis vector: a pilot study. Scand J Med Sci Sports 26:809-815. https://doi. org/10.1111/sms.12514

Neville J, Wixted A, Rowlands D, James D (2010) Accelerometers: an underutilized resource in sports monitoring. In: 2010 Sixth international conference on intelligent sensors, sensor networks and information processing. IEEE, pp 287-290

Oh K, Park S (2017) The bending stiffness of shoes is beneficial to running energetics if it does not disturb the natural MTP joint flexion. J Biomech 53:127-135. https://doi.org/10.1016/j.jbiom ech.2017.01.014

Pataky TC, Robinson MA, Vanrenterghem J (2013) Vector field statistical analysis of kinematic and force trajectories. J Biomech 46:2394-2401. https://doi.org/10.1016/j.jbiomech.2013.07.031

Pataky TC, Vanrenterghem J, Robinson MA (2015) Zero- vs. onedimensional, parametric vs. non-parametric, and confidence interval vs. hypothesis testing procedures in one-dimensional biomechanical trajectory analysis. J Biomech 48:1277-1285. https ://doi.org/10.1016/j.jbiomech.2015.02.051

Roy J-PR, Stefanyshyn DJ (2006) Shoe midsole longitudinal bending stiffness and running economy, joint energy, and EMG. Med Sci Sport Exerc 38:562-569. https://doi.org/10.1249/01.mss.00001 93562.22001.e8

Saunders PU, Pyne DB, Telford RD, Hawley JA (2004) Factors affecting running economy in trained distance runners. Sports Med 34:465-485

Shorten M, Mientjes MIV (2011) The 'heel impact' force peak during running is neither 'heel' nor 'impact' and does not quantify shoe cushioning effects. Footwear Sci 3:41-58

Sinclair J, Mcgrath R, Brook O, Taylor PJ, Dillon S (2016) Influence of footwear designed to boost energy return on running economy in comparison to a conventional running shoe. J Sports Sci 34:10941098. https://doi.org/10.1080/02640414.2015.1088961

Sousa CV, Sales MM, Nikolaidis PT, Rosemann T, Knechtle B (2018) How much further for the sub-2-hour marathon? Open Access J Sport Med 9:139-145. https://doi.org/10.2147/OAJSM.S169758

Weir JB de (1949) New methods for calculating metabolic rate with special reference to protein metabolism. J Physiol 109:1-9

Willwacher S, König M, Potthast W, Brüggemann G-P (2013) Does specific footwear facilitate energy storage and return at the metatarsophalangeal joint in running? J Appl Biomech 29:583-592

Willwacher S, König M, Braunstein B, Goldmann J-P, Brüggemann G-P (2014) The gearing function of running shoe longitudinal bending stiffness. Gait Posture 40:386-390. https://doi. org/10.1016/j.gaitpost.2014.05.005

Worobets J, Wannop JW, Tomaras E, Stefanyshyn D (2014) Softer and more resilient running shoe cushioning properties enhance running economy. Footwear Sci 6:147-153. https://doi. org/10.1080/19424280.2014.918184 